EPJ Web of Conferences 49, 02001 (2013)

DOI: $10.1051 /$ epjconf/20134902001

(C) Owned by the authors, published by EDP Sciences, 2013

\title{
Heavy-Ion Physics in a Nutshell
}

\author{
Tetsufumi Hirano ${ }^{1, a}$ \\ ${ }^{1}$ Depatment of Physics, Sophia University, Tokyo 102-8554, Japan
}

\begin{abstract}
The physics of quark gluon plasma (QGP) and heavy ion collisions at the collider energies is briefly reviewed. We first discuss about the discovery of a nearly perfect fluidity of the QGP. We also highlights recent topics on responses of the QGP to initial deformation and propagation of a jet.
\end{abstract}

\section{Introduction}

The standard theory of strong interaction is quantum chromodynamics (QCD) which governs dynamics of color charged particles/fields such as quarks and gluons. Like photons in quantum electrodynamics, gluons are gauge bosons which mediate the strong force among matter particles (namely quarks in QCD). Contrary to photons which do not have electric charges, gluons carry color charges and consequently interact among themselves. This kind of self-interaction is manifestation of QCD as a non-Abelian gauge theory. It was shown that the coupling "constant" among color charges decreases with increasing energy scale in non-Abelian gauge theory $[1,2]$. Then one can expect that quarks and gluons, which are ordinary confined into hadrons at low energies, are freed at extremely high temperature. The novel matter in which the quarks and gluons are thermally equilibrated is called the quark gluon plasma (QGP) [3].

Let us suppose that a sort of transition from a hadron gas to the QGP takes place when the lightest hadrons, pions, are close-packed. The number density of closepacked system is

$$
n_{\pi}=v_{\pi}^{-1}, \quad v_{\pi}=\frac{4 \pi r_{\pi}^{3}}{3},
$$

where $r_{\pi} \sim 0.5 \mathrm{fm}\left(=5 \times 10^{-16} \mathrm{~m}\right)$. On the other hand, let us further suppose that pions obey the equation of state of a relativistic ideal gas. Thus, the number density becomes

$$
n_{\pi} \approx 3.6 T^{3}
$$

where $T$ is temperature of the gas. From Eqs. (1) and (2), transition temperature can be estimated as $\sim 100$ $\mathrm{MeV} / k_{B} \approx 10^{12} \mathrm{~K}$. Although estimation above is the simplest enough, the first principle calculation based on lattice QCD [4] also gives the same order of the transition temperature $\sim 160 \mathrm{MeV} / k_{B}$ : Energy density rapidly increases around the temperature and the quark/gluon degree of freedom inside hadrons are "activated" above this temperature.

\footnotetext{
ae-mail: hirano@sophia.ac.jp
}

In a more strict sense, susceptibility of the chiral order parameter has a peak around this, but does not diverge. Thus the two phases are not clearly separated. Such a continuous change is called "cross-over".

The matter at such an extremely high temperature existed up to $\sim 10$ micro second after the Big Bang. In this era, the universe was filled with the QGP. There is a unique opportunity to investigate such an extreme matter even on the Earth using heavy-ion reactions at relativistic energies: By accelerating heavy nuclei almost at the speed of light and colliding them with each other, kinetic energy of colliding nuclei could turn into thermal energy. The matter created in this way can exist only for a very short time $\left(\sim 10 \mathrm{fm} / c \approx 10^{-23} \mathrm{sec}\right)$. Unlike experiments in the condensed matter physics, one cannot control thermodynamic variables or even lifetime of the system in these experiments. One measures at most the final particle distributions originated from the created matter and tries to find traces of the QGP in them. The main purpose of the physics of relativistic heavy ion collisions is to pursue the properties of the QGP through these experimental results. Heavy ion programs at Large Hadron Collider (LHC) in CERN and at Relativistic Heavy Ion Collider (RHIC) in Brookhaven National Laboratory have been running currently to explore the QGP.

As of the time writing this article, temperature measured at RHIC was recorded as the highest man-made temperature by the Guinness World Records [5]. PHENIX Collaboration measured photon spectra and deduced the temperature of created matter as $T \sim 220 \mathrm{MeV} / k_{B}$ [6]. It should be noted that new photon data at LHC break this "world's record" very recently and the measured maximum temperature is estimated as $T \sim 300 \mathrm{MeV} / k_{B}$ [7].

In this paper, the physics of the QGP and relativistic heavy ion collisions is briefly reviewed. Responses of the QGP to initial deformation and jet propagation are also discussed. 


\section{QGP as a Nearly Perfect Fluid}

Since the existence of the QGP was deduced from the asymptotic freedom as discussed in the Introduction, the interaction among quarks and gluons was supposed to be weak. If this picture was valid, the QGP would have behaved like a gas and its dynamics would have been described by kinetic theory. On the other hand, what was discovered at RHIC [8] exhibits totally opposite behaviour: it turned out that hydrodynamic description of the QGP works reasonably well, which indicates discovery of a nearly perfect fluidity of the QGP and, in turn, strong coupling nature of quarks and gluons in the QGP [9-14]. One of the key observables is azimuthally anisotropic distribution, namely, the second order harmonics of the distribution, also known as elliptic flow [15], $v_{2}=\langle\cos (2 \phi)\rangle$. Here $\phi$ is azimuthal angle measured from the reaction plane and $\langle\cdots\rangle$ is an average weighting azimuthal distribution of produced particles. $v_{2}$ is sensitive to degree of thermalisation and hydrodynamic property of the matter. Thus this is an ideal probe of produced matter in relativistic heavy ion collisions.

Relativistic hydrodynamic equation describes energy momentum conservation

$$
\begin{aligned}
\partial_{\mu} T^{\mu \nu} & =0, \\
T^{\mu \nu} & =e u^{\mu} u^{\nu}-(P+\Pi) \Delta^{\mu \nu}+\pi^{\mu \nu} .
\end{aligned}
$$

Here $T^{\mu v}$ is energy momentum tensor, $e$ is energy density and $P$ is hydrostatic pressure. $\Pi$ is bulk pressure and $\pi^{\mu v}$ is shear stress tensor, both of which are dissipative quantities. $u^{\mu}$ is four flow velocity and $\Delta^{\mu \nu}=g^{\mu \nu}-u^{\mu} u^{\nu}$ is a projection tensor perpendicular to $u^{\mu}$. The above equation requires the equation of state, $P=P(e)$, in ideal hydrodynamic simulations. In addition, constitutive equations (i.e., relations between dissipative currents and thermodynamic forces) and transport coefficients such as shear viscosity and bulk viscosity are needed in the case of viscous hydrodynamics simulations.

Early ideal hydrodynamic calculations using the Glauber model initialisation and neglecting dissipative quantities were successful in reproduction of $v_{2}$ data at RHIC [16-21]. After the discovery, a main focus in the hydrodynamic analysis was put on constraint of transport coefficients, in particular, the ratio of shear viscosity to entropy density, $\eta / s$, using viscous hydrodynamic simulations. It should be noted that it is not a trivial task to implement viscosity in relativistic hydrodynamics since a naive extension of Navier-Stokes type hydrodynamic equations to its relativistic version violates causality [22]. To remedy this issue, the relaxation effect on dissipative currents plays an important role in the formulation of causal hydrodynamics and has to be taken into account in constitutive equations [23-25]. The effect has already been implemented in recent viscous hydrodynamic simulations to constrain the transport coefficients [26-31]. The ratio, $\eta / s$, is found to be $\sim 0.08-0.16$ through an analysis using a cutting-edge hybrid model which combines viscous hydrodynamic description of the QGP fluid with kinetic description of the hadron gas $[30,31]$. It is interesting to mention that $\eta / s$ for water is $\sim 400$ time larger than this value.
There still exists an uncertainty of the ratio which comes from relatively unknown initial state of the high-energy heavy ion reaction [32]. Understanding of pre-equilibrium stage is one of the longstanding problems in relativistic heavy ion collisions.

\section{Highlights from Recent Topics}

In this section, we pick up recent intriguing phenomena at RHIC and LHC and discuss about them from a theoretical point of view. We first show the QGP response to fluctuation of initial profiles which result in higher order harmonics of azimuthal distributions. We next describe propagation of an energetic parton inside the QGP which wakes intriguing patterns of flow.

\subsection{QGP Response to Initial Fluctuation}

Transverse position of nucleons inside a nucleus is distributed according to the Woods-Saxon nuclear density profile. Collisions between two nucleons inside colliding two nuclei happen randomly in the overlap area in a single event. Thus, the shape of initial QGP fluctuates from event to event.

To quantify the deformation of the QGP, one defines the $n$-th order of deformation parameter as

$$
\varepsilon_{n}=\frac{\left|\left\langle r^{2} e^{i n \phi}\right\rangle\right|}{\left\langle r^{2}\right\rangle},
$$

where $r$ is a distance from center of "mass", $(\langle x\rangle,\langle y\rangle), \phi$ is azimuthal angle and $\langle\cdots\rangle$ denotes an average weighting an initial profile of, e.g., energy density distribution or entropy density distribution.

The system responds to initial deformation of the QGP profile in the configuration space. This can be measured through, e.g., two-particle correlation function ${ }^{1}$

$$
C(\Delta \phi) \approx 1+2 \sum_{n=1}^{\infty} v_{n}\left(p_{1}\right) v_{n}\left(p_{2}\right) \cos n \Delta \phi .
$$

Figure 1 shows $v_{n}$ as a function of the order $n$ [36] in ultracentral $\mathrm{Pb}+\mathrm{Pb}$ collisions at $\mathrm{LHC}$, which are compared with results from recent state-of-the-art viscous hydrodynamic simulations from two independent groups. By changing the ratio of shear viscosity to entropy density $\eta / s$, they calculated higher order anisotropic flow $v_{n}$ in top $0-0.2 \%$ central $\mathrm{Pb}+\mathrm{Pb}$ collisions at $\sqrt{s_{N N}}=2.76 \mathrm{TeV}$. Since the corresponding impact parameters are tiny for these events, initial deformation contains solely fluctuating effects. Figure 1 (left) shows a comparison of the CMS data with results from Luzum and Ollitrault [34]. Hydrodynamic results are obtained by using the Glauber model initialisation. $v_{n}$ results with $\eta / s \sim 0.2$ are comparable with the CMS data. In Fig. 1 (right), hydrodynamic results from Shen, Qiu and Heinz [35] are compared with the CMS data. A larger $\eta / s$ in the Kharzeev-Levin-Nardi model initialisation is needed to reproduce the CMS data than in the Glauber model initialisation.

\footnotetext{
${ }^{1}$ There are various experimental analysis methods to obtain $v_{n}$. See, for example, Ref. [33].
} 

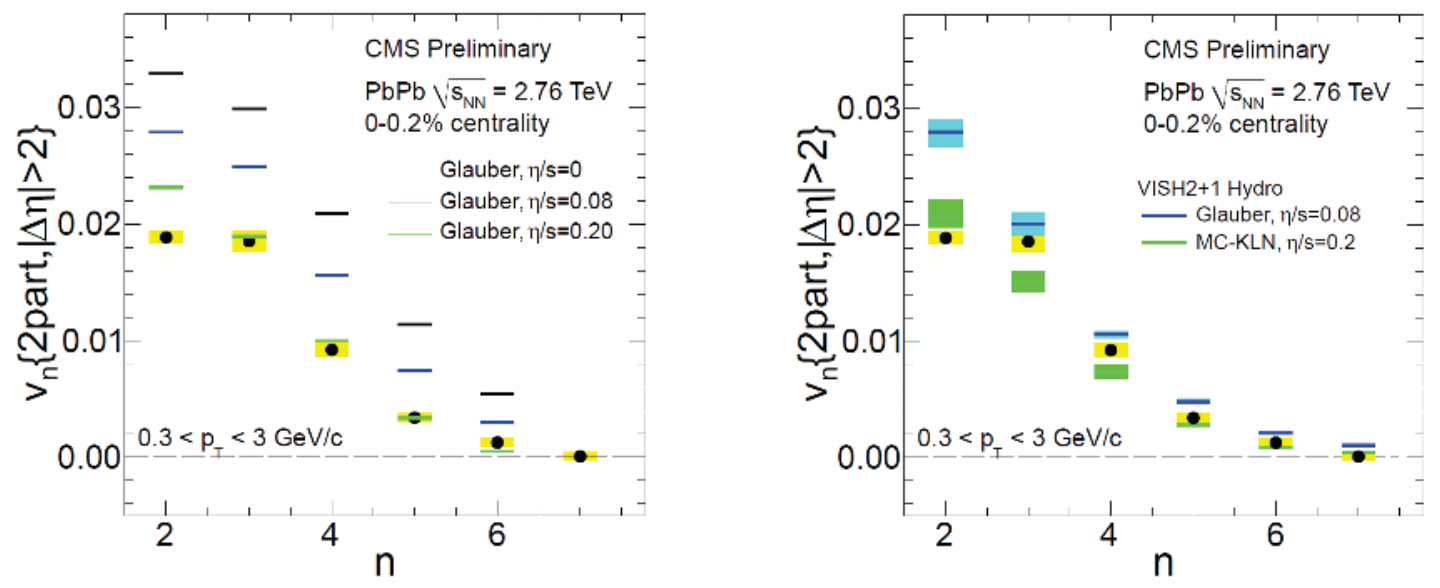

Figure 1. $v_{n}$ versus $n$ in ultra-central $(0-0.2 \%) \mathrm{Pb}+\mathrm{Pb}$ collisions at $\sqrt{s_{N N}}=2.76 \mathrm{TeV}$ obtained by the CMS Collaboration are compared with hydrodynamic results. Figures are taken from Ref. [36].

These data remind us an analysis of cosmic microwave background $[37,38]$. Similar to this, higher order coefficients correspond to small structure of the system, which might be related with small coarse graining size. There have been several analyses on flow data from this point of view [39-45].

\subsection{QGP Response to Jet Propagation}

Jets and mini-jets are produced in relativistic heavy ion collisions at the collider energies. Contrary to protonproton collisions, these energetic partons are subject to traverse QGP medium created at the same time. During traveling medium, these partons lose their energies through interaction between the parton and the medium. In general, this is called jet quenching [46]. When path length of two jets differs from each other, this results in an imbalanced jet pair. Apparently, energy and momentum are not balanced in some events. A natural question would be "Where do these lost energies go?" One of the clues to answer this question is distribution of particles out of the jet cones.

Figure 2 shows a sum of transverse momenta projected onto the sub-leading jet axis

$$
p_{T}^{\|}=\sum_{i}-p_{T}^{i} \cos \left(\phi_{i}-\phi_{\mathrm{LJA}}\right)
$$

as a function of asymmetric parameter

$$
A_{J}=\frac{p_{T, 1}-p_{T, 2}}{p_{T, 1}+p_{T, 2}}
$$

from the CMS Collaboration [47]. Here $p_{T, 1}>120 \mathrm{GeV} / c$ and $p_{T, 2}>50 \mathrm{GeV} / c$ are, respectively, transverse momentum of leading and sub-leading jets. $p_{T}^{i}$ and $\phi_{i}$ are transverse momentum and azimuthal angle for each particle, respectively. $\phi_{\mathrm{LJA}}$ is azimuthal angle of leading jet axis. Figure 2 (left) shows that total momentum parallel to the jet axis is balanced among large $(p>8 \mathrm{GeV} / c)$ momentum particles in the leading jet direction and small $(p<8$
$\mathrm{GeV} / c$ ) momentum particles in the sub-leading (opposite) direction. It turns out that transverse momentum along the jet axis is balanced between leading particles with high $p_{T}$ inside the jet cone and low momentum particles distributed over a wide angular range from the sub-leading jet axis, which is shown in Fig. 2 (right). This implies that low momentum particles at large angle from the jet axis carry lost energies from the jet traversing medium.

It is not well understood how the lost energy is distributed among low momentum particles. To study the response of the QGP fluid to propagation of a jets, we use relativistic hydrodynamic equations with source terms [48]

$$
\partial_{\mu} T_{\text {fluid }}^{\mu v}(x)=J^{v}(x) .
$$

Here $T_{\text {fluid }}^{\mu \nu}$ is the energy momentum tensor of the QGP fluid and $J^{v}$ is the density of the four momentum deposited from the traversing jets. We assume that the deposited energy and momentum are instantaneously thermalised inside a fluid cell. For simplicity, we consider collisional energy loss only. When a massless jet partcle travels through the QGP fluid, the source term is given by

$$
\begin{aligned}
J^{0}(x) & =-\frac{d p_{\text {jet }}^{0}}{d t} \delta^{(3)}\left(\boldsymbol{x}-\boldsymbol{x}_{\text {jet }}(t)\right), \\
\boldsymbol{J}(x) & =\frac{\boldsymbol{p}_{\text {jet }}}{p_{\text {jet }}^{0}} J^{0}(x) .
\end{aligned}
$$

We solve Eq. (9) numerically without linearisation. In this way, we describe collective flow induced by jets on top of an expanding background QGP fluid in high-energy heavy-ion collisions.

We consider two different situations. As a test case, we consider one jet particle traveling through a uniform fluid. As a more realistic case, we consider a pair of jets traveling in opposite directions through an expanding fluid. In both cases, we consider a perfect fluid in full $(3+1)$ dimensional space and use massless ideal gas equation of state $P=e / 3$. We assume that jet particles are massless and travel in a straight line. 

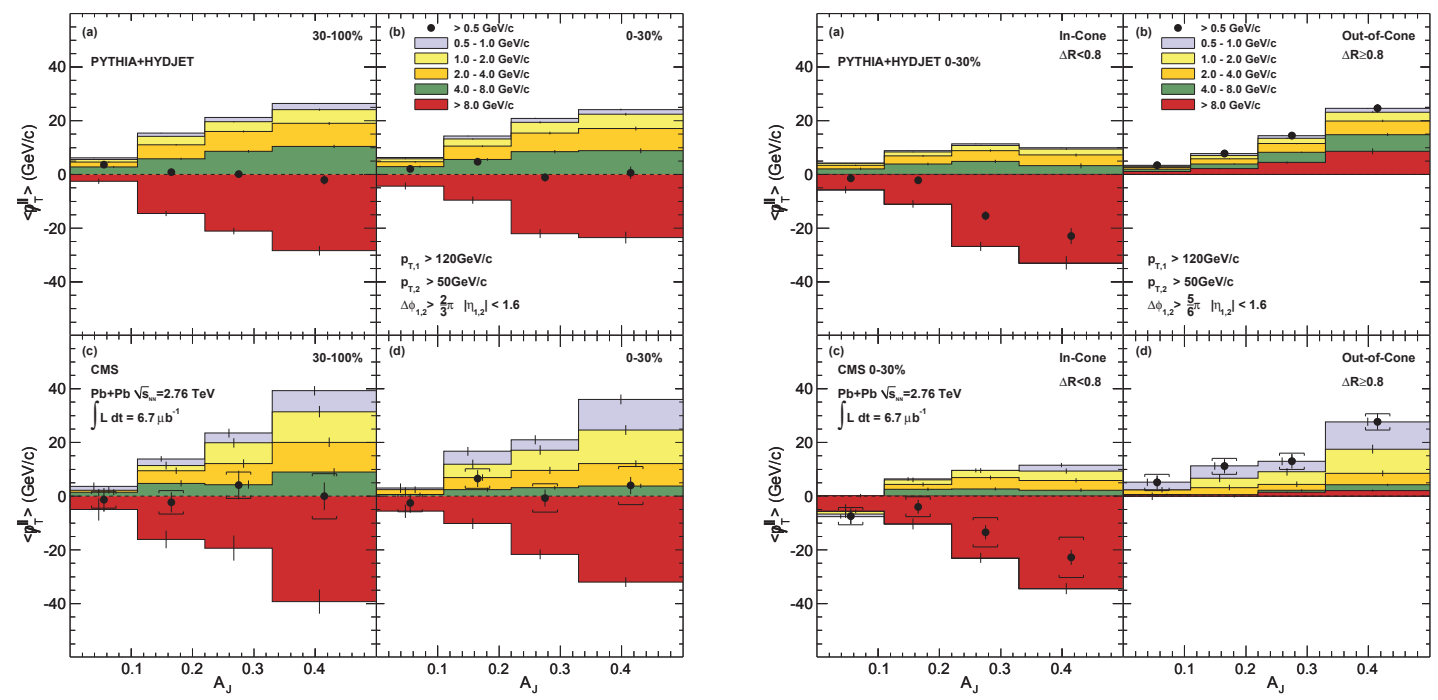

Figure 2. The top and bottom rows show results for PYTHIA+HYDJET and PbPb data, respectively. Left: $p_{T}^{\|}$for particles with $p_{T}>$ $0.5 \mathrm{GeV} / c$ projected onto the leading jet axis (solid circles) for 30-100\% centrality (a) and (c) and 0-30\% centrality (b) and (d). Right: $p_{T}^{\|}$for $0-30 \%$ centrality, inside $(\Delta R<0.8)$ one of the leading or sub-leading jet cones (a) and (c) and outside $(\Delta R>0.8)$ the leading and sub-leading jet cones (b) and (d). Figures are taken from Ref. [47].

Figure 3 (left) shows the energy density distribution and the flow velocity field of the QGP fluid in a plane including a jet axis at a fixed time $t=9 \mathrm{fm} / c$. Initial energy density of the fluid is $0.019 \mathrm{GeV}^{4}$. Propagation of the jet particle starts in the $x$-direction from the origin at the speed of light at $t=0 \mathrm{fm} / c$. One sees the Mach cone, namely a "V-shaped" cone-like structure of higher energy density region in three dimensional space. The energy density inside the cone is lower than that of the backgrounds. On the surface of the cone, flow velocity is nearly perpendicular to the surface. The jet particle is followed by a strong collective flow in the positive $x$ direction. Behind the cone, one sees vortices around $(x, y)=(5 \mathrm{fm}, \pm 2 \mathrm{fm})$ which form a vortex ring around the passage of the jet in three dimensional space.

Next, we consider a pair of jets traveling through a QGP fluid which expands radially. Initial energy density distribution is

$$
e(t=0, \boldsymbol{x})=\left\{\begin{array}{cc}
\frac{3 T_{0}^{4}}{\pi^{2}} \exp \left(-\frac{\boldsymbol{x}^{2}}{2 \sigma^{2}}\right), & (|\boldsymbol{x}| \leq R) \\
0 . & (|\boldsymbol{x}|>R)
\end{array}\right.
$$

Here, we choose $T_{0}=0.5 \mathrm{GeV}, \sigma=1.5 \mathrm{fm}$ and $R=6 \mathrm{fm}$ as a typical parameter set for central heavy collisions at the LHC energy. Without jets, the fluid expands isotropically. A pair of jets is supposed to be created at an off-central position $(x, y, z)=(3 \mathrm{fm}, 0 \mathrm{fm}, 0 \mathrm{fm})$ with the common initial energy $150 \mathrm{GeV}$, both of which are traveling in the opposite direction. One jet goes in the positive $x$ direction and is to be observed as a leading jet. The other goes in the negative $x$ direction, loses more energy and is to be observed as a sub-leading jet.
To see the momentum acquired by the QGP fluid from the jets, we calculate

$$
\Delta\left(\frac{d\left\langle p^{\|}\right\rangle_{i}}{d \cos \theta}\right)=\sum_{p \in i} \frac{d p^{\|}}{d \cos \theta}-\left.\sum_{p \in i} \frac{d p^{\|}}{d \cos \theta}\right|_{\text {no jet }},
$$

where $\theta$ is the polar angle from the leading jet, $p$ the momentum of the particle from the fluid, and $i$ the index of the momentum regions. Summation of the particles is taken over in the momentum region: high-momentum ( $p>$ $8 \mathrm{GeV})$, intermediate momentum $(4 \mathrm{GeV}<p<8 \mathrm{GeV})$ or low momentum $(0 \mathrm{GeV}<p<4 \mathrm{GeV})$. We calculate the deposited momentum distribution, Eq. (13), through the Cooper-Frye formula [49] at a fixed time and do not consider jets' momentum. Figure 3 (right) shows the deposited momentum distribution as a function of $\cos \theta$. $\cos \theta=-1$ corresponds to the direction of the sub-leading jet. A prominent peak appears in this direction for each momentum region. At large angles from the sub-leading jet $(\cos \theta>-0.94)$, low momentum particles give dominant contribution. This result is qualitatively consistent with the results shown in Fig. 2 [47]. The distribution of deposited momentum would be sensitive to transport properties of the QGP as well as energy loss mechanism of a jet traversing the medium.

\section{Summary}

We first discussed the discovery of a nearly perfect fluidity of the QGP in relativistic heavy ion collisions and the latest results for the ratio of shear viscosity to entropy density, $\eta / s$ from hydrodynamic analyses. We also discussed the QGP response to initial deformation as a new channel to constrain the $\eta / s$. Finally we investigated a possible interpretation of the dijet events in relativistic heavy ion collisions using relativistic hydrodynamics with a source term. 

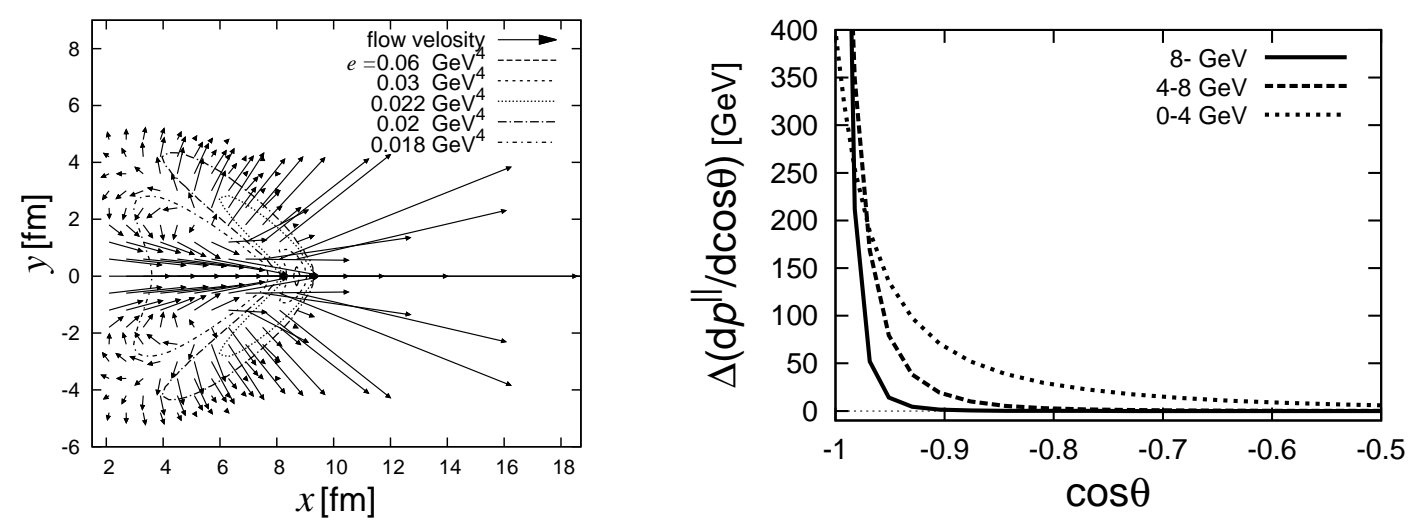

Figure 3. Left: Energy density contours and flow velocity vectors around the jet axis at $t=9 \mathrm{fm} / c$. A jet particle is located at $(x, y)=(9$ $\mathrm{fm}, 0 \mathrm{fm}$ ). Background energy density is $0.019 \mathrm{GeV}^{4}$. Right: Deposited momentum distribution as a function of polar angle measured from the leading jet direction. Figures are taken from Ref. [48]

We showed low momentum particle emission at wide angular range can result from a Mach cone structure due to energy loss of an energetic parton on top of the isotropically expanding medium.

\section{References}

[1] D. J. Gross and F. Wilczek, Phys. Rev. Lett. 30, 1343 (1973).

[2] H. D. Politzer, Phys. Rev. Lett. 30, 1346 (1973).

[3] K. Yagi, T. Hatsuda and Y. Miake, Camb. Monogr. Part. Phys. Nucl. Phys. Cosmol. 23, 1 (2005).

[4] S. Borsanyi, G. Endrodi, Z. Fodor, A. Jakovac, S. D. Katz, S. Krieg, C. Ratti and K. K. Szabo, JHEP 1011, 077 (2010).

[5] http://www.guinnessworldrecords.com/ world-records/10000/highest-man-made-temperature

[6] A. Adare et al. [PHENIX Collaboration], Phys. Rev. Lett. 104, 132301 (2010)

[7] R. Preghenella [ALICE Collaboration], these proceedings.

[8] http://www.bnl.gov/newsroom/news.php?a=1303

[9] U. Heinz and P. F. Kolb, Nucl. Phys. A 702, 269 (2002).

[10] M. Gyulassy, preprint arXiv:nucl-th/0403032

[11] T. D. Lee, Nucl. Phys. A 750, 1 (2005).

[12] M. Gyulassy, L. McLerran, Nucl. Phys. A 750, 30 (2005).

[13] E. V. Shuryak, Nucl. Phys. A 750, 64 (2005).

[14] T. Hirano and M. Gyulassy, Nucl. Phys. A 769, 71 (2006).

[15] J. Y. Ollitrault, Phys. Rev. D 46, 229 (1992).

[16] P. F. Kolb, P. Huovinen, U. W. Heinz and H. Heiselberg, Phys. Lett. B 500, 232 (2001).

[17] P. Huovinen, P. F. Kolb, U. W. Heinz, P. V. Ruuskanen and S. A. Voloshin, Phys. Lett. B 503, 58 (2001).

[18] D. Teaney, J. Lauret and E. V. Shuryak, Phys. Rev. Lett. 86, 4783 (2001);
[19] D. Teaney, J. Lauret and E. V. Shuryak, preprint arXiv:nucl-th/0110037

[20] T. Hirano, Phys. Rev. C 65, 011901 (2002).

[21] T. Hirano and K. Tsuda, Phys. Rev. C 66, 054905 (2002).

[22] W.A. Hiscock and L. Lindblom, Phys. Rev. D 31, 725 (1985).

[23] I. Müller, Z. Phys. 198, 329 (1967).

[24] W. Israel, Annals Phys. 100, 310 (1976).

[25] W. Israel and J. M. Stewart, Annals Phys. 118, 341 (1979).

[26] P. Romatschke and U. Romatschke, Phys. Rev. Lett. 99, 172301 (2007).

[27] M. Luzum and P. Romatschke, Phys. Rev. C 78, 034915 (2008).

[28] K. Dusling and D. Teaney, Phys. Rev. C 77, 034905 (2008).

[29] H. Song and U. W. Heinz, Phys. Rev. C 77, 064901 (2008).

[30] H. Song, S. A. Bass, U. Heinz, T. Hirano and C. Shen, Phys. Rev. Lett. 106, 192301 (2011) [Erratumibid. 109, 139904 (2012)].

[31] H. Song, S. A. Bass, U. Heinz, T. Hirano and C. Shen, Phys. Rev. C 83, 054910 (2011).

[32] T. Hirano, U. W. Heinz, D. Kharzeev, R. Lacey and Y. Nara, Phys. Lett. B 636, 299 (2006).

[33] S. A. Voloshin, A. M. Poskanzer and R. Snellings, arXiv:0809.2949 [nucl-ex].

[34] M. Luzum and J. -Y. Ollitrault, arXiv:1210.6010 [nucl-th].

[35] Z. Qiu, C. Shen and U. Heinz, Phys. Lett. B 707, 151 (2012).

[36] CMS Collaboration, CMS-PAS-HIN-12-011.

[37] E. Komatsu et al. [WMAP Collaboration], Astrophys. J. Suppl. 180 (2009) 330

[38] http://lambda.gsfc.nasa.gov/toolbox/

[39] P. Sorensen, arXiv:0808.0503 [nucl-ex].

[40] A. P. Mishra, R. K. Mohapatra, P. S. Saumia and A. M. Srivastava, Phys. Rev. C 81, 034903 (2010). 
[41] E. Shuryak, Phys. Rev. C 80, 054908 (2009). [Erratum-ibid. C 80, 069902 (2009).]

[42] P. Staig and E. Shuryak, Phys. Rev. C 84, 034908 (2011).

[43] P. Staig and E. Shuryak, Phys. Rev. C 84, 044912 (2011).

[44] A. Mocsy and P. Sorensen, preprint arXiv:1008.3381 [hep-ph]
[45] P. Sorensen, B. Bolliet, A. Mocsy, Y. Pandit and N. Pruthi, Phys. Lett. B 705, 71 (2011).

[46] See, for example, D. d'Enterria and B. Betz, Lect. Notes Phys. 785, 285 (2010).

[47] S. Chatrchyan et al. [CMS Collaboration], Phys. Rev. C 84, 024906 (2011).

[48] Y. Tachibana and T. Hirano, arXiv:1210.5567 [nuclth].

[49] F. Cooper and G. Frye, Phys. Rev. D 10, 186 (1974). 